\title{
Time-of-use price optimizing model and its solving method
}

\author{
Guosheng ZHAO ${ }^{1,}$, , Tianle $\mathrm{ZHAN}^{1, \mathrm{~b}}$,Hongguang $\mathrm{XI}^{1, \mathrm{c}}$ \\ ${ }^{1}$ School of Electrical Engineering, Zhengzhou University, Zhengzhou 450001, China

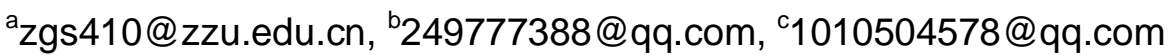

\begin{abstract}
Keywords:time-of-use price;price elasticity matrix of demand;multi-objective optimization model;ideal point method

Abstract:Time-of-use (TOU) price can reduce load of power grids fluctuation effectively and increase the efficiency of electric power system operation. The key to the successful implementation of TOU price is the establishment of TOU price. The TOU price optimization model is based on price elasticity matrix of demand.The optimizing model is a non-linear multiple objects optimal model and it is solved by ideal point method.At last it is proved by example that TOU price optimization model can avoid the peak load and it is also feasibility.
\end{abstract}

\section{Introduction}

TOU price is the most widely-implemented economic instruments in all economic instruments of power demand side management ${ }^{[1-3]}$, now most of industrial and commercial customers have already used TOU price ,in some provinces,residents have also used TOU price.For the user of implementing TOU price, at peak load period electricity price is higher,at valley load period electricity price is lower.TOU price can guide users to transfer load from peak load period to valley load period and reduce load fluctuation.

The key to the successful implementation of TOU price is the establishment of TOU price.At present there are a lot of literatures that research how to set TOU price,for example ,literature [4] analyses consumer's respondence to the price and establish TOU price optimizing model which is based on multi-agent system simulation,literature [5] analyses that after the implementation of TOU price,electricity consumption habits and electricity expenditure of users will change and user satisfaction index will be affected and establishs TOU price optimizing mode which consider users reaction and satisfaction index,literature [6] analyses the interests of the power supply and establish TOU price optimizing mode based on the interests of the power supply.

This paper constructs TOU price optimizing model based on price elasticity matrix of demand.The optimizing model is a non-linear multiple objects optimal model,in order to let all objective functions obtain ideal value,this paper uses ideal point method to solve the model.At last the result shows that the optimizing model and solving method is effective by simulation.

\section{Price elasticity matrix of demand}

The consumer's electricity consumption is directly affected by electricity price,so to set electricity price profile reasonable needs research how electricity consumers adjust their electricity demand according to the changes of electricity sales price. In this paper we define that electricity price elasticity coefficients is the relative variation of power consumption the relative variation of electricity charges within one load operation cycle:

$$
e=\frac{\Delta q}{q}\left(\frac{\Delta p}{p}\right)^{-1}
$$

where, $e$ is electricity price elasticity coefficients, $q$ isoriginal load, $\Delta q$ is variation of load, $p$ is original electricity price, $\Delta p$ is the variation of electricity price.

According to consumer psychology, the consumer will transfer load from the time when electricity price is high to others, so the energy consumption quantity is affected not only by current price, but also by adjacent prices.In order to obtain energy consumption quantity in different periods, we use 
self-elasticity coefficient and cross-elasticity coefficient to describe the relationship between energy consumption quantity and electricity price in different periods.Price elasticity matrix of demand is made of self-elasticity coefficient and cross-elasticity coefficient.In our country,TOU price divides one day into three periods,so we can get:

$$
\begin{aligned}
& {\left[\begin{array}{c}
\frac{Q_{1}^{\prime}-Q_{1}}{Q_{1}} \\
\frac{Q_{2}^{\prime}-Q_{2}}{Q_{2}} \\
\frac{Q_{3}^{\prime}-Q_{3}}{Q_{3}}
\end{array}\right]=E\left[\begin{array}{c}
\frac{p_{1}-p_{0}}{p_{0}} \\
\frac{p_{2}-p_{0}}{p_{0}} \\
\frac{p_{m}-p_{0}}{p_{0}}
\end{array}\right]} \\
& E=\left[\begin{array}{cccc}
e_{11} & e_{12} & \mathrm{~L} & e_{1 m} \\
e_{21} & e_{22} & \mathrm{~L} & e_{2 m} \\
\mathrm{M} & \mathrm{M} & & \mathrm{M} \\
e_{m 1} & e_{m 2} & \mathrm{~L} & e_{m m}
\end{array}\right]
\end{aligned}
$$

Where $Q_{i}$ is load before the implementation of TOU price, $Q_{i}^{\prime}$ is load after the implementation of TOU price, $p_{0}$ is electricity price before the implementation of TOU price, $p_{i}$ is electricity price after the implementation of TOU price,here $i=1,2,3$ is peak time period, flat time period,valley time period。 $E$ is price elasticity matrix of demand, $e_{i i}$ is self-elasticity coefficient, $e_{i j}$ is cross-elasticity coefficient 。

\section{Time-of-use price optimizing model and its solving method}

\section{Time-of-use price optimizing mode}

1)the constraint condition of customer's interest

After the implementation of TOU price, in order to get support from consumers, consumers need get real benefit,the average of electricity price for consumers should be reduced:

$$
\frac{p_{1} Q_{1}^{\prime}+p_{2} Q_{2}^{\prime}+p_{3} Q_{3}^{\prime}}{Q_{1}^{\prime}+Q_{2}+Q_{3}}<p_{0}
$$

2)the constraint condition of power supply company's interest

The implementation of TOU price can improve efficiency of power grid operation,save the investment and reduce the operating costs of power supply company,so the electric selling revenue of power supply company's can decrease slightly:

$$
(1-M)\left(p_{0}-p_{m}\right)\left(Q_{1}+Q_{2}+Q_{3}\right) \leq p_{1} Q_{1}^{\prime}+p_{2} Q_{2}^{\prime}+p_{3} Q_{3}^{\prime}-p_{m}\left(Q_{1}^{\prime}+Q_{2}^{\prime}+Q_{3}^{\prime}\right)
$$

Where $M$ is the maximum ratio of the loss of power supply company revenue, $p_{m}$ is average of power price.

3)the constraint condition of peak-valley price

If the ratio of peak electricity price and valley electricity price is too low, the effect of peak load shifting is poor, if the ratio of peak electricity price and valley electricity price is too high, the mode of production and living habits of consumers will be affected,so according to actual condition,we can get: 


$$
\left\{\begin{array}{l}
1.3 \leq \frac{P_{1}}{P_{0}} \leq 1.8 \\
0.8 \leq \frac{P_{2}}{P_{0}} \leq 1.2 \\
0.4 \leq \frac{P_{3}}{P_{0}} \leq 0.8
\end{array}\right.
$$

4)the constraint condition of the total load of consumers

After the implementation of TOU price,the total load of consumers should remain substantially retained,in order to keep power system stability and meet the need of consumers' production and living :

$$
1-\delta \leq \frac{Q_{1}^{\prime}+Q_{2}^{\prime}+Q_{3}^{\prime}}{Q_{1}+Q_{2}+Q_{3}} \leq 1+\delta
$$

Where $\delta$ is allowable range of the total load of consumers

\section{the objective functions of time-of-use price optimizing model}

The main aim of TOU price is to avoid the peak load and reduce peak-valley difference,so the objective functions of time-of-use price optimizing model can be described as follows:

1) Minimize peak load

$$
\min \tau=\min \max q_{i} \quad i=1,2,3 \ldots 24
$$

2)Minimize peak-valley difference

$$
\min \omega=\min \left(\max q_{i}-\min q_{j}\right) i, j=1,2,3 \ldots 24
$$

\section{solving method of the time-of-use price optimizing model}

The optimization model is a non-linear multiple objects optimal mode,the solving method which most of literatures use is based on the weighted average value of different goals, but that the weighted value is subjective and it will affect the objectivity of the results.In order to avoid subjective influence, in this paper we use ideal point method to solve the optimization model.Ideal point method considers different objective functions as the coordinates of a point,so objective functions can form a target point.First we can use fmincon function in matlab to get optimal values of every single objective,then these optimal values of every single objective can form a ideal point,at last a new objective function which is based on the Euclidean distance of these two points can be constructed .Ideal point method can taking into account all the objective functions, The detailed solution process is as shown as follows:

The value of different objective functions has a large difference,so first calculate the maximum and minimum of every single objective: $\tau_{\max } 、 \tau_{\min } 、 v_{\max } 、 v_{\min }$, then normalize the two objective functions:

$$
\begin{aligned}
& \bar{\tau}=\frac{\tau-\tau_{\min }}{\tau_{\text {max }}-\tau_{\text {min }}} \\
& \bar{v}=\frac{v-v_{\text {min }}}{v_{\text {max }}-v_{\text {min }}}
\end{aligned}
$$

The target point is $(\bar{\tau}, \bar{v})$, the ideal value of $\bar{\tau}$ and $\bar{v}$ is 0 , so the ideal point is $(0,0)$. According to ideal point method,we can construct a new objetive function:

$$
\min f=\min \sqrt{\bar{\tau}^{2}+\bar{v}^{2}}
$$




\section{Numerical example}

Typical daily load in a certain place in Guangdong Province before the implementation of TOU price is shown as follows:

Tab.1 Typical daily load

\begin{tabular}{cccccccc}
\hline $\mathrm{t}$ & $\begin{array}{c}\text { Load } \\
(\mathrm{MW})\end{array}$ & $\mathrm{t}$ & $\begin{array}{c}\text { Load } \\
(\mathrm{MW})\end{array}$ & $\mathrm{t}$ & $\begin{array}{c}\text { Load } \\
(\mathrm{MW})\end{array}$ & $\mathrm{t}$ & $\begin{array}{c}\text { Load } \\
(\mathrm{MW})\end{array}$ \\
\hline 1 & 168 & 7 & 171 & 13 & 192 & 19 & 213 \\
2 & 164 & 8 & 183 & 14 & 216 & 20 & 215 \\
3 & 160 & 9 & 188 & 15 & 233 & 21 & 222 \\
4 & 162 & 10 & 191 & 16 & 238 & 22 & 205 \\
5 & 158 & 11 & 195 & 17 & 196 & 23 & 192 \\
6 & 165 & 12 & 198 & 18 & 186 & 24 & 170 \\
\hline
\end{tabular}

The electricity price before the implementation of TOU price is $0.527 \mathrm{yuan} / \mathrm{kwh}$, the average pool purchase price is 0.4 yuan/kwh.Peak and valley time periods are partitioned as follows:Peak time period contains 14:00 17:00 and $19: 00 \sim 22: 00$;Flat time period contains $8: 00 \sim 14: 00,17: 00 \sim$ $19: 00$ and $22: 00 \sim 24: 00$; Valley time period contains $24: 00 \sim 8: 00$.In this paper,we suppose the maximum ratio of Power supply company's interest concession $M=0.05$, the maximum ratio of the change of the total load of consumers $\delta=0.02$.Literature[7] scientifically analyzes calculation method of price elasticity matrix of demand and get its value by example,so in this paper we use the value that literature[7] gets:

$$
E=\left[\begin{array}{ccc}
-0.1732 & 0.1333 & 0.1066 \\
0.1495 & -0.1150 & 0.0923 \\
0.2045 & 0.1650 & -0.1320
\end{array}\right]
$$

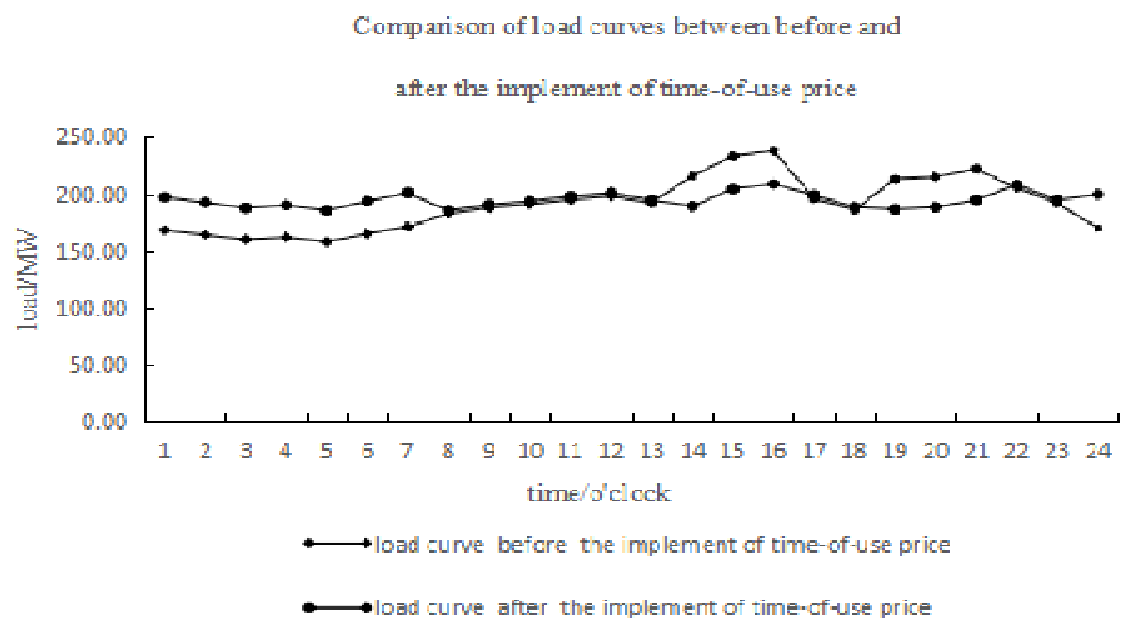

Figure.1 Comparison of load curves between before and after the implement of time-of-use price

Before the implementation of TOU price,the value of peak load is maximum $: \tau_{\max }=238$, the value of peak-valley difference is maximum: $v_{\max }=80$, after the implementation of TOU price, we can obtain the the minimum value of peak load: $\tau_{\min }=208.5860$ and the the minimum value of peak-valley difference: $v_{\min }=23.1442$, then according to ideal point method,we can obain that when peak electricity price is $0.7715 \mathrm{yuan} / \mathrm{kwh}$, flat electricity price is $0.5684 \mathrm{yuan} / \mathrm{kwh}$, valley electricity price is 0.2631 yuan $/ \mathrm{kwh}$ the objective functions obtain the optimal value, in this case, the value of peak load is $208.6709 \mathrm{MW}$, the value of peak-valley difference is $23.1904 \mathrm{MW}$. 


\section{Conclusion}

The implementation of TOU price can reduce the fluctuation of load in power system and increase the efficiency of electric power system operation, The key to the successful impleentation of TOU price is the establishment of TOU price.This paper constructs time-of-use price optimizing model according to the characteristic of TOU price and price elasticity matrix of demand.The optimizing model which is a non-linear multiple objects optimal model,in this paper, we use ideal point method to solve the optimal model.At last,we prove that the time-of-use price optimizing model and its solving method in this paper is reasonable by example.

\section{References}

[1] Zhou Ming,Li Gengyin,Ni Yixin.A prelimInary research on implementation mechanism of demand side management underelectricity market[J].Power System Technology.2005,29(5):611.

[2] Wang Beibei,Li Yang,Gao Ciwei.Demand side management outlook under smart grid infrastructure[J].Automation of Electric Power Systems,2009,33(20):17-22.

[3] Tang Mozhu.Research and application of smart grid demand side management system[J].Iournal of Electric Power,2011,26(4):322-324.

[4] Tan Jinjing,Wang Beibei,LiYang.Modeling of user response to time-of-use price based on multi agent technology[J]. Power System Technology,2012,6(2):257-263.

[5] Ding Wei,Yuan Jiahai,Hu Zhaoguang.Time-of-use price decisionmodel considering users reaction and satisfaction index[J]. Automation of Electric Power Systems, 2005, 29(20):10-14.

[6] Guo Lianzhe,Tan Zhongfu,Li Xiaojun.Demand response based model and method for optimal design of time-of-use electricity price[J].,2006,30(5):24-28.

[7] Chen Cangyang,Hu Bo,Xie Kaigui,Wan Lingyun,Xiang Bin.A peak-valley tou price model considering power system reliability and power purchase risk[J].Power System Technology,2014,38(8):2041-2048.2005,29(5):6-11. 